\title{
Superfluidity and effective mass of magnetoexcitons in topological insulator bilayers: Effect of inter-Landau-level Coulomb interaction
}

\author{
Zhigang Wang, ${ }^{1}$ Zhen-Guo Fu, ${ }^{2,1}$ and Ping Zhang1,, \\ ${ }^{1}$ LCP, Institute of Applied Physics and Computational Mathematics, \\ P.O. Box 8009, Beijing 100088, People's Republic of China \\ ${ }^{2}$ State Key Laboratory for Superlattices and Microstructures, \\ Institute of Semiconductors, Chinese Academy of Sciences, \\ P. O. Box 912, Beijing 100083, People's Republic of China
}

\begin{abstract}
The effective mass and superfluidity-normal phase transition temperature of magnetoexcitons in topological insulator bilayers are theoretically investigated. The intra-Landau-level Coulomb interaction is treated perturbatively, from which the effective magnetoexciton mass is analytically discussed. The inclusion of inter-Landau-level Coulomb interaction by more exact numerical diagonalization of the Hamiltonian brings out important modifications to magnetoexciton properties, which are specially characterized by prominent reduction in the magnetoexciton effective mass and promotion in the superfluidity-normal phase transition temperature at a wide range of external parameters.

PACS numbers: 73.21.Ac, 73.22.Pr, 73.30.+y
\end{abstract}

\section{INTRODUCTION}

The bilayer $n-p$ systems [1, 2], comprising electrons from the $n$ layer and holes from the $p$ layer, have been the subject of recent theoretical and experimental investigations. These systems, including coupled quantum wells [3-6] and layered graphene [7-13], are of interest, in particular, in connection with the possibility of the Bose-Einstein condensation and superfluidity of indirect excitons or electron-hole pairs. The superfluidity is manifested as nondissipative flow of electric currents, equal in magnitude and opposite in direction, along the layers. In high magnetic fields, two-dimensional excitons survive in a substantially wider temperature range, as the exciton binding energies increase with magnetic field.

Recently, topological insulator (TI) as a new phase of quantum matter, which can not be adiabatically connected to conventional insulators and semiconductors, have been studied intensively [14 23]. Present technological advances have allowed the production of topological insulator bilayers (TIBs) system, which consist of two TI thin films separated by a dielectric barrier. On one hand, $\mathrm{CdTe} / \mathrm{HgTe}$ TI quantum wells are two-dimensional which are ideal for designing the TIBs, since $\mathrm{CdTe} / \mathrm{HgTe}$ can be either doped in $n$-type or $p$-type [24]; while on the other hand, although the three-dimensional $\mathrm{TI} \mathrm{Bi}_{2} \mathrm{Se}_{3}$ is dominated by charged selenium vacancies, which results in $n$-type behavior, it could also exhibit $p$-type behavior by doping method. Transport and magnetic properties of TIs doped by $\mathrm{Cr}, \mathrm{Fe}$, and $\mathrm{Cu}$, and $n$-type to $p$-type crossover in $\mathrm{Bi}_{2} \mathrm{Se}_{3}$ codoped with $\mathrm{Sb}$ and $\mathrm{Pb}$ have been investigated variously in experiments. Recently, in particular, the $p$-type $\mathrm{Bi}_{2} \mathrm{Se}_{3}$ single crystal has been ob-

\footnotetext{
*Corresponding author; zhang-ping@iapcm.ac.cn
}

tained by doping $\mathrm{Ca}$ in experiments [25]. It is possible to use $\mathrm{Al}_{2} \mathrm{O}_{3}$ or $\mathrm{SiO}_{2}$ as the dielectric spacer, on which, remarkably, high-quality $\mathrm{Bi}_{2} \mathrm{Se}_{3}$ and $\mathrm{Bi}_{2} \mathrm{Te}_{3}$ TI quantum well thin films have been now successively grown 26 29]. Besides, high quality $\mathrm{Bi}_{2} \mathrm{Se}_{3} / \mathrm{ZnSe}$ multilayers [30], and superlattices constructed by alternating $\mathrm{Bi}_{2} \mathrm{Se}_{3}$ and $\mathrm{In}_{2} \mathrm{Se}_{3}$ layers [31] have also been successfully obtained in experiments.

TIs are characterized by a full insulating gap in the bulk and protected gapless edge or surface states. It is the gapless edge or surface states that results in the absence of exciton at the TI heterostructures. However, there are various methods, such as introduction of a magnetic exchange field or a strong external magnetic field, to produce a gap in TI heterostructures, which may lead to the formation of excitons [32, 33] or magnetoexcitons. Magnetoexcitons, which have been observed experimentally in semiconductor quantum wells, is an ideal object in exploring the Coulomb interaction effects in TIBs system and Bose-Einstein condensation since it behaves as neutral bosons at low densities. Moreover, as mentioned above, the TIBs, which are required for generating magnetoexcitons, are achievable in current experimental capabilities. Motivated by its importance both from basic point of interest and from application of TI-based electronics, in the present paper we address this issue by presenting an attempt at the theoretical evaluation of superfluidity property and effective mass of magnetoexcitons in the TIBs structure [see Fig. 1(a)] in the presence of a perpendicular high magnetic field, which produces a gap since the Dirac-type energy spectrum becomes discrete by forming Landau levels (LLs).

In the presence of the high magnetic field, the system may exhibit the phase transition from the hightemperature disordered or delocalized phase with the exponential correlation to a low-temperature quasi-ordered or localized phase, which is named as Kosterlitz-Thouless (KT) transition. We study the properties of magnetoex- 


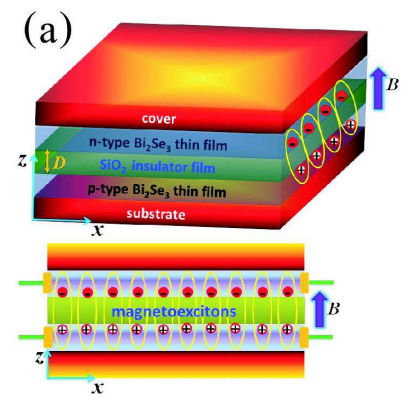

(b)

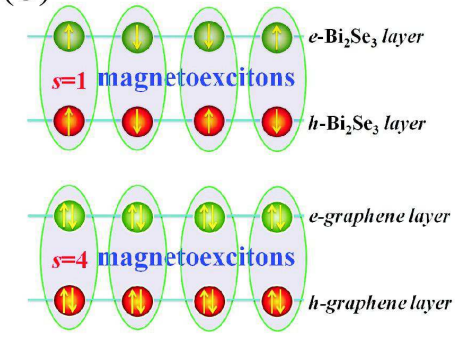

FIG. 1: (Color online) (a) Scheme of TIB comprising two $\mathrm{Bi}_{2} \mathrm{Se}_{3}$ thin films separated by $\mathrm{SiO}_{2}$ spacer. Electron (hole) carriers are induced by $n(p)$-type doping or applied gates. To produce magnetoexcitons a strong perpendicular magnetic field is applied. (b) Illustration of the difference of magnetoexcitons between TIB and graphene bilayer. The spin degeneracy factor $s=4$ in graphene bilayer leads its undressed magnetoexciton mass to be $1 / 4$ times of that in TIB.

citons in TIBs, including the effective magnetoexciton mass $m_{B}$ and superfluid-normal state, i.e., KT transition temperature $T_{c}$. Comparing to bilayer graphene [11], the effective mass of magnetoexcitons in TIBs is found to be four times larger due to the spin degeneration [see Fig. 1(b)]. Meanwhile, we also investigate the effect of inter-LL Coulomb interaction on the magnetoexciton superfluidity in TIBs, which has been ignored in previous studies on semiconductor and graphene systems. We find that although the intra-LL Coulomb interaction plays the main role in deciding the effective magnetoexciton mass and KT temperature, the inclusion of inter-LL Coulomb interaction brings out important modifications. In fact, at the magnetic field $B=10 \sim 20 \mathrm{~T}$ and the spacer thickness $D=25 \sim 35 \mathrm{~nm}$ in the setup in Fig. 1(a), our results show that the inter-LL Coulomb interaction will reduce the magnetoexciton effective mass by $15 \% \sim 25 \%$ and improve the KT temperature by $20 \% \sim 25 \%$.

\section{EFFECTIVE MAGNETIC MASS}

We start with the effective Hamiltonian of TIB system $H=H_{0}+U(r)$, where

$$
H_{0}=v_{F} \sigma_{e} \cdot\left(\hat{\mathbf{z}} \times \pi_{e}\right)-v_{F} \sigma_{h} \cdot\left(\hat{\mathbf{z}} \times \pi_{h}\right)
$$

is the free electron-hole part of TIB, while $U(r)=$ $-e^{2} / \epsilon \sqrt{|\mathbf{r}|^{2}+D^{2}}$ is the Coulomb interaction between a pair of electron and hole with $r=r_{e}-r_{h}$ being the relative coordinate in the $x-y$ plane and $D$ the spacer thickness. Here, $r_{e(h)}$ represents the position of the electron (hole), and $\pi_{e(h)}=p_{e(h)} \pm e A_{e(h)} / c=-i \partial / \partial r_{e(h)} \pm$ $e A_{e(h)} / c$ denotes the in-plane momentum of the electron (hole), where the gauge is chosen as $A_{e(h)}=B\left(0, x_{e(h)}, 0\right)$ in the following calculations. $v_{F}$ is the Fermi velocity $\left(\sim 3 \times 10^{5} \mathrm{~m} / \mathrm{s}\right.$ for $\mathrm{Bi}_{2} \mathrm{Se}_{3}$-family materials $), \hat{z}$ is the unit vector normal to the surface, and $\sigma_{e}=\sigma \otimes I\left(\sigma_{h}=I\right.$ $\otimes \sigma)$ describes the spin operator acting on the electron (hole), in which $\sigma$ denotes a vector of Pauli matrices and $I$ is the $2 \times 2$ identity matrix.

The LLs of $H_{0}$ are given by

$$
E_{n_{+}, n_{-}}^{(0)}=\frac{\sqrt{2} v_{F}}{r_{B}}\left[\operatorname{sgn}\left(n_{+}\right) \sqrt{\left|n_{+}\right|}-\operatorname{sgn}\left(n_{-}\right) \sqrt{\left|n_{-}\right|}\right] .
$$

The corresponding eigenstates can be analytically expressed as [10]

$$
\psi_{\mathbf{P}}(\mathbf{R}, \mathbf{r})=\exp \left[i\left(\mathbf{P}+\frac{e}{2 c}[\mathbf{B} \times \mathbf{r}]\right) \cdot \mathbf{R}\right] \Psi\left(\mathbf{r}-\mathbf{r}_{0}\right),
$$

where $R=\left(r_{e}+r_{h}\right) / 2$ and $r_{0}=r_{B}^{2}(\hat{B} \times P)$ with magnetic length $r_{B}=\sqrt{\hbar c / e B}$ and unit magnetic field $\hat{B}$. For an electron in LL $n_{+}$and a hole in LL $n_{-}$, the fourcomponent wave functions in the relative coordinate are written as

$$
\begin{aligned}
& \Psi_{n_{+}, n_{-}}(\mathbf{r})=\left|n_{+}, n_{-}\right\rangle \\
& =(\sqrt{2})^{\delta_{n_{+}, 0}+\delta_{n_{-}, 0}-2}\left(\begin{array}{c}
\Phi_{\left|n_{+}\right|-1,\left|n_{-}\right|-1}(\mathbf{r}) \\
i^{-\operatorname{sgn}\left(n_{-}\right)} \Phi_{\left|n_{+}\right|-1,\left|n_{-}\right|}(\mathbf{r}) \\
i^{\operatorname{sgn}\left(n_{+}\right)} \Phi_{\left|n_{+}\right|,\left|n_{-}\right|-1}(\mathbf{r}) \\
i^{\operatorname{sgn}\left(n_{+}\right)-\operatorname{sgn}\left(n_{-}\right)} \Phi_{\left|n_{+}\right|,\left|n_{-}\right|}(\mathbf{r})
\end{array}\right),
\end{aligned}
$$

where

$$
\begin{aligned}
\Phi_{n_{1}, n_{2}}(\mathbf{r}) & =\frac{2^{-\frac{\left|l_{z}\right|}{2}}}{\sqrt{2 \pi}} \frac{n_{-} !}{\sqrt{n_{1} ! n_{2} !}} \frac{1}{r_{B}} \\
& \times e^{-i l_{z} \phi} \operatorname{sgn}\left(l_{z}\right)^{l_{z}} \frac{r^{\left|l_{z}\right|}}{r_{B}^{\left|l_{z}\right|}} L_{n_{-}}^{\left|l_{z}\right|}\left(r^{2} / 2 r_{B}^{2}\right) e^{-r^{2} / 4 r_{B}^{2}}
\end{aligned}
$$

with $L$ denoting Laguerre polynomials $z=x+i y, l_{z}=$ $n_{1}-n_{2}, n_{-}=\min \left(n_{1}, n_{2}\right), z /|z|=e^{i \phi}$, and $\operatorname{sgn}\left(l_{z}\right)^{l_{z}} \rightarrow 1$ for $l_{z}=0$.

When the inter-LL Coulomb interaction is taken into account, however, one should perform diagonalization of full Hamiltonian of Coulomb interacting carriers in some basis of magnetoexcitonic states $\Psi_{n_{+}, n_{-}}(r)$. In other words, to obtain eigenvalues of the Hamiltonian $H$, we need to numerically solve the following eigen-equation [34]:

$$
\begin{aligned}
0 & =\operatorname{det} \| \delta_{n_{+}, n_{+}^{\prime}} \delta_{n_{-}, n_{-}^{\prime}}\left(E_{n_{+}, n_{-}}^{(0)}-E\right) \\
& +\left\langle\Psi_{n_{+}^{\prime}, n_{-}^{\prime}}\left|U\left(\mathbf{r}-r_{B}^{2} \hat{\mathbf{z}} \times \mathbf{P}\right)\right| \Psi_{n_{+}, n_{-}}\right\rangle \| .
\end{aligned}
$$

The location of the chemical potential will determine the possible LL indices for electrons and holes. We should point out that the intra-LL component of the Coulomb interaction is defined as $\left\langle\Psi_{n_{+}, n_{-}}|U| \Psi_{n_{+}, n_{-}}\right\rangle$, while the inter-LL component is defined as $\left\langle\Psi_{n_{+}^{\prime}, n_{-}^{\prime}}|U| \Psi_{n_{+}, n_{-}}\right\rangle$, where $\left|\Psi_{n_{+}^{\prime}, n_{-}^{\prime}}\right\rangle \neq\left|\Psi_{n_{+}, n_{-}}\right\rangle$.

Consider a magnetoexciton is formed by an electron on the LL $n$ and a hole on the LL $m$. In the limit of the relatively large separation $D$ between electron and 

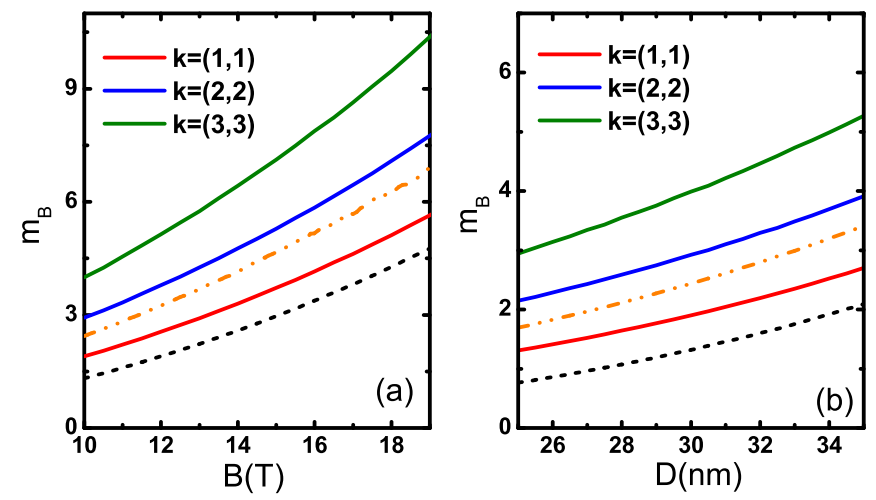

FIG. 2: (Color online) Calculated magnetoexciton effective mass $m_{B}$ (in unit of free electron mass) as functions of (a) magnetic field $B$ and (b) spacer width $D$, for different choices of magnetoexciton LL indices $k=(n, n)$. For comparison, the $k=(1,1)$ magnetoexciton effective mass without including inter-LL transition (given by Eq. (11)) is plotted by dashdot-dotted lines and $m_{B}^{(0)}(D)$ given by Eq. (12) is shown by dashed lines. The spacer width in panel (a) is set as $D=30$ $\mathrm{nm}$ and the external magnetic field in panel (b) is set as $B=10$ $\mathrm{T}$ in (b). The other parameters are set as $v_{F}=3.0 \times 10^{5} \mathrm{~m} / \mathrm{s}$ and $\epsilon=4.0$.

hole TIBs and relatively high magnetic field $B$ when $e^{2} /(\epsilon D) \ll \hbar v_{F} / r_{B}$, the magnetoexciton energy can be approximated by only considering its zeroth order energy part $E_{n, m}^{(0)}$. However, at higher magnetic field $10 \sim 20 \mathrm{~T}$ in experiment, the Coulomb interaction $e^{2} /(\epsilon D)$ is only several times less than the zeroth energy $\hbar v_{F} / r_{B}$. In this case, the Coulomb interaction can be treated as a perturbation, which could be departed into intra-LL part and inter-LL part. For the intra-LL part, the magnetoexciton energy can be written as

$$
\begin{aligned}
E_{n, m} & =E_{n, m}^{(0)}+\left\langle\Psi_{n, m}\left|U\left(\mathbf{r}-r_{B}^{2} \hat{\mathbf{z}} \times \mathbf{P}\right)\right| \Psi_{n, m}\right\rangle \\
& =E_{n, m}^{(0)}+2^{\delta_{n, 0}+\delta_{m, 0}-2} \\
& \times\{\langle\langle|n|-1,|m|-1, \mathbf{P}|U||n|-1,|m|-1, \mathbf{P}\rangle\rangle \\
& +\langle\langle|n|-1,|m|, \mathbf{P}|U||n|-1,|m|, \mathbf{P}\rangle\rangle \\
& +\langle\langle|n|,|m|-1, \mathbf{P}|U||n|,|m|-1, \mathbf{P}\rangle\rangle \\
& +\langle\langle|n|,|m|, \mathbf{P}|U||n|,|m|, \mathbf{P}\rangle\rangle\}
\end{aligned}
$$

where the notation $\langle\langle n m P|U| n m P\rangle\rangle$ denotes the averaging by the two-dimensional harmonic oscillator eigenfunctions $\Phi_{n, m}(r)$. Substituting for small magnetic momenta $P \ll \hbar / r_{B}$ and $P \ll \hbar D / r_{B}^{2}$ the relation

$$
\langle\langle n m P|U| n m P\rangle\rangle=\mathcal{E}_{n m}^{(b)}+\frac{P^{2}}{2 M_{n m}(B, D)}
$$

into Eq. (77), we can get the dispersion law of a magnetoexciton for small magnetic momenta. As an example, let us consider the magnetoexciton on the $k=(1,1)$ LL. The magnetoexciton energy at small magnetic momenta is then given by

$$
E_{1,1}(P)=\mathcal{E}_{B}^{(b)}(D)+\frac{P^{2}}{2 m_{B}(D)},
$$

where the binding energy $E_{B}^{(b)}(D)$ is expressed as

$$
\mathcal{E}_{B}^{(b)}(D)=\frac{1}{4} E_{00}^{(b)}+\frac{1}{2} E_{01}^{(b)}+\frac{1}{4} \mathcal{E}_{11}^{(b)}
$$

and the effective magnetic mass $m_{B}(D)$ of a magnetoexciton is expressed as

$$
\frac{1}{m_{B}}=\frac{1}{4 M_{00}}+\frac{1}{2 M_{01}}+\frac{1}{4 M_{11}} .
$$

Here, the constants $E_{00}^{(b)}, E_{01}^{(b)}, E_{11}^{(b)}, M_{00}, M_{01}$, and $M_{11}$ depend on magnetic field $B$ and the interlayer separation $D$. Explicitly, $E_{00}^{(b)}=-C E_{0}, E_{01}^{(b)}=-E_{0}\left[\left(\frac{1}{2}-x\right) C+\frac{x}{\sqrt{\pi}}\right]$, $E_{11}^{(b)}=-E_{0}\left[\left(\frac{3}{4}+x^{2}+x^{4}\right) C-\frac{1}{\sqrt{\pi}}\left(\frac{x}{2}+x^{3}\right)\right]$, and $M_{00}=$ $M_{0}\left[\left(1+2 x^{2}\right) C-\frac{2 x}{\sqrt{\pi}}\right]^{-1}, M_{01}=M_{0}\left[\left(3+2 x^{2}\right) \frac{x}{\sqrt{\pi}}-\left(\frac{1}{2}+\right.\right.$ $\left.\left.4 x^{2}+2 x^{4}\right) C\right]^{-1}, M_{11}=M_{0}\left[\frac{C}{4}\left(7+50 x^{2}+44 x^{4}+8 x^{6}\right)-\right.$ $\left.\left(\frac{17}{2}+10 x^{2}+2 x^{4}\right) \frac{x}{\sqrt{\pi}}\right]^{-1}$, with $x=D /\left(\sqrt{2} r_{B}\right), E_{0}=$ $\frac{e^{2}}{\epsilon r_{B}} \sqrt{\frac{\pi}{2}}, M_{0}=\frac{2^{3 / 2} \epsilon \hbar^{2}}{\sqrt{\pi} e^{2} r_{B}}$, and functions $\operatorname{erfc}(x)$ and $C(x)$ respectively defined as $\operatorname{erfc}(x)=\frac{2}{\sqrt{\pi}} \int_{x}^{+\infty} \exp \left(-t^{2}\right) d t$ and $C(x)=e^{x^{2}} \operatorname{erfc}(x)$. In the special limit of $D \gg r_{B}$, one obtains

$$
m_{B}^{(0)}(D)=\frac{D^{3} \hbar^{2} \epsilon}{r_{B}^{4} e^{2}}
$$

We should notice that the effective magnetic mass in the present system is equal to that in coupled semiconductor quantum wells at the same $D, \epsilon$, and $B$, which is four times larger than that in graphene bilayers [11, 13]. Although the effective low-energy Hamiltonian of the electron-hole graphene bilayer system is also expressed as a $4 \times 4$ matrix, which is similar to Eq. (11) shown above, the momentum therein is actually coupled with the pseudospins of sublattices rather than the real spins of electron and hole. Therefore, the factor $1 / 4$ in the effective magnetic mass of magnetoexcitons in graphene bilayer system arises from the spin degeneracy (see Fig. $1(\mathrm{~b})$ ), which is absent in the TIB system considered here since the freedom of spin has been involved due to the strong spin-orbit coupling in the original effective Hamiltonian Eq. (11). This significant difference between TIB and graphene bilayer may be detected by the gyromagnetic resonance experiments.

Now let us make a simple estimate to illustrate the reasonability of the perturbation theory. We choose the Fermi velocity and the electron-hole interlayer distance as $v_{F}=3 \times 10^{5} \mathrm{~m} / \mathrm{s}$ and $D=30 \mathrm{~nm}$. The dielectric constant of the spacer $\mathrm{SiO}_{2}$ is $\epsilon=4.0$. Under these conditions, one can obtain the Coulomb interaction $e^{2} /(\epsilon D)=12 \mathrm{meV}$ and kinetic energy $\hbar v_{F} / r_{B}=24.4 \mathrm{meV}$ (corresponding to $r_{B}=8.1 \mathrm{~nm}$ ) when the applied magnetic field $B=10 \mathrm{~T}$. 

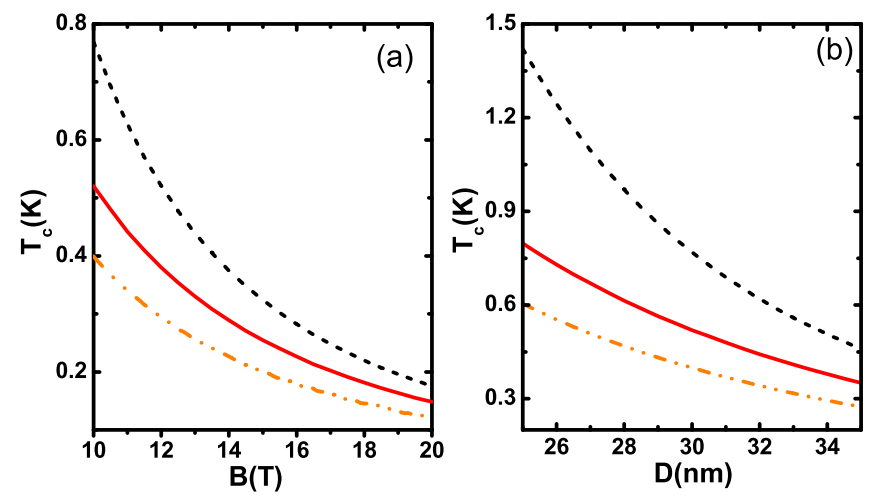

FIG. 3: (Color online) Calculated KT temperature versus (a) magnetic field and (b) spacer width. The density of magnetoexciton is set as $n=4.0 \times 10^{11} \mathrm{~cm}^{-2}$. The spacer width $D=30 \mathrm{~nm}$ in used in panel (a) and the magnetic field $B=10$ $\mathrm{T}$ is used in panel (b). The red solid, black dashed, and blue dotted lines correspond to the magnetoexciton effective mass obtained by considering both intra-LL and inter-LL Coulomb interaction, only intra-LL Coulomb interaction, and the approximation Eq. (12) at $D \gg r_{B}$, respectively.

In this case, $e^{2} /(\epsilon D) /\left(\hbar v_{F} / r_{B}\right) \simeq 0.5$, which indicates that in our studied TIB system, the effect of Coulomb interaction can be treated as a perturbation. Same analysis can also be applied to graphene, in which the Fermi velocity is about 3 times larger than that in $\mathrm{Bi}_{2} \mathrm{Se}_{3}$ film. With the same external parameters for estimation, one can immediately obtain $e^{2} /(\epsilon D) /\left(\hbar v_{F} / r_{B}\right) \simeq 0.17$ for graphene bilayer system. In the above calculations of the magnetoexciton energy and effective mass we only consider the intra-LL Coulomb interaction. On the other hand, the inter-LL Coulomb interaction is also important. We will show in the following that the inter-LL Coulomb interaction can bring about $0.15 \sim 0.25$ times modification of the magnetoexciton effective mass (see Fig. 2).

To study the whole effect of Coulomb interaction, including the intra-LL and inter-LL Coulomb interaction, on the effective magnetoexciton mass as well as KT transition to the superfluid state, we have to numerically solve Eq. (6). In our numerical calculations we have used four electron and four hole levels around the LL $k=(n, n)$. Figures 2(a) and 2(b) respectively show the numerically calculated magnetoexciton mass $m_{B}(D)$ as a function of the magnetic field and the dielectric spacer width $D$ with different LL indices $k$. To observe the effect of inter-LL Coulomb interaction, we also exhibit in both figures the magnetoexciton effective mass at LL $k=(1,1)$ by dashdotted lines without inter-LL transition [Eq. (11)], and the analytical approximation results of magnetoexciton mass $m_{B}^{(0)}$ [Eq. (12)] by dashed lines. Comparing these results, one can find that for the magnetic field strength $B=10 \sim 20$ T and the spacer width $D=25 \sim 35 \mathrm{~nm}$, the magnetoexciton effective mass is reduced by $15 \% \sim 25 \%$ by the inclusion of the inter-LL Coulomb interaction.

\section{SUPERFLUIDITY OF DIPOLE MAGNETOEXCITONS}

The magnetoexcitons that are constructed by spatially separated electrons and holes in TIB at large interlayer separation $D \gg r_{B}$ form two-dimensional weakly nonideal gas of bosons. This is valid, because we can think the indirect exciton interact as parallel dipoles when $D \gg r_{B}$. So at low temperatures, these magnetoexcitons condense in Bose-Einstein type and form superfluid state below the KT transition temperature $T_{c}$ [35, 36], which is determined by

$$
T_{c}=\frac{\pi \hbar^{2} n_{s}\left(T_{c}\right)}{2 k_{B} m_{B}},
$$

where $n_{s}(T)$ is the superfluid density of the magnetoexciton system and $k_{B}$ is the Boltzmann constant.

To solve the superfluid density $n_{s}$ in Eq. (13), we can first solve the normal density $n_{n}$ because $n_{s}=n-n_{n}$, where $n$ is the total density. Through the usual procedure [11, 37], we have the normal density $n_{n}$ as

$$
n_{n}=\frac{3 \zeta(3)}{2 \pi \hbar^{2}} \frac{k_{B}^{3} T^{3}}{m_{B} c_{s}^{4}}
$$

where $\zeta(z)$ is the Riemann zeta function and $c_{s}=$ $\sqrt{\mu / m_{B}}$ is the sound velocity with the chemical potential

$$
\mu=\frac{\pi \hbar^{2} n}{m_{B} \ln \left[\hbar^{4} \epsilon^{2} /\left(2 \pi n m_{B}^{2} e^{4} D^{4}\right)\right]} .
$$

Finally, combining Eqs. (13)-15), we obtain the KT transition temperature

$$
\begin{aligned}
T_{c} & =\left[\left(1+\sqrt{\frac{32}{27}\left(\frac{m_{B} k_{B} T_{c}^{0}}{\pi \hbar^{2} n}\right)^{3}+1}\right)^{1 / 3}\right. \\
& \left.-\left(\sqrt{\frac{32}{27}\left(\frac{m_{B} k_{B} T_{c}^{0}}{\pi \hbar^{2} n}\right)^{3}+1}-1\right)^{1 / 3}\right] \frac{T_{c}^{0}}{2^{1 / 3}},
\end{aligned}
$$

where $T_{c}^{0}=\frac{1}{k_{B}}\left(\frac{2 \pi \hbar^{2} n \mu^{2}}{3 m_{B} \zeta(3)}\right)^{1 / 3}$ is an auxiliary quantity that is equal to the temperature at which the superfluid density vanishes in the mean-field approximation (i.e., $\left.n_{s}\left(T_{c}^{0}\right)=0\right)$.

We calculate $T_{c}$ for different choices of magnetic field $(10 \sim 20 \mathrm{~T})$, dielectric spacer width $D(25 \sim 35 \mathrm{~nm})$, and magnetoexciton density $n$ (up to $1.0 \times 10^{12} \mathrm{~cm}^{-2}$ ). The results are presented in Fig. 3 and Fig. 4. One can find the following features: (i) The KT temperature is up to several Kelvins in our calculated ranges, which is similar to that in graphene bilayer and coupled semiconductor quantum wells [11, 12]; (ii) Figure 3 clearly shows that the calculated $T_{c}$ evidently depends on the effective magnetoexciton mass form. Comparing the $T_{c}$ calculated with full Coulomb interaction (red lines) with that in the 


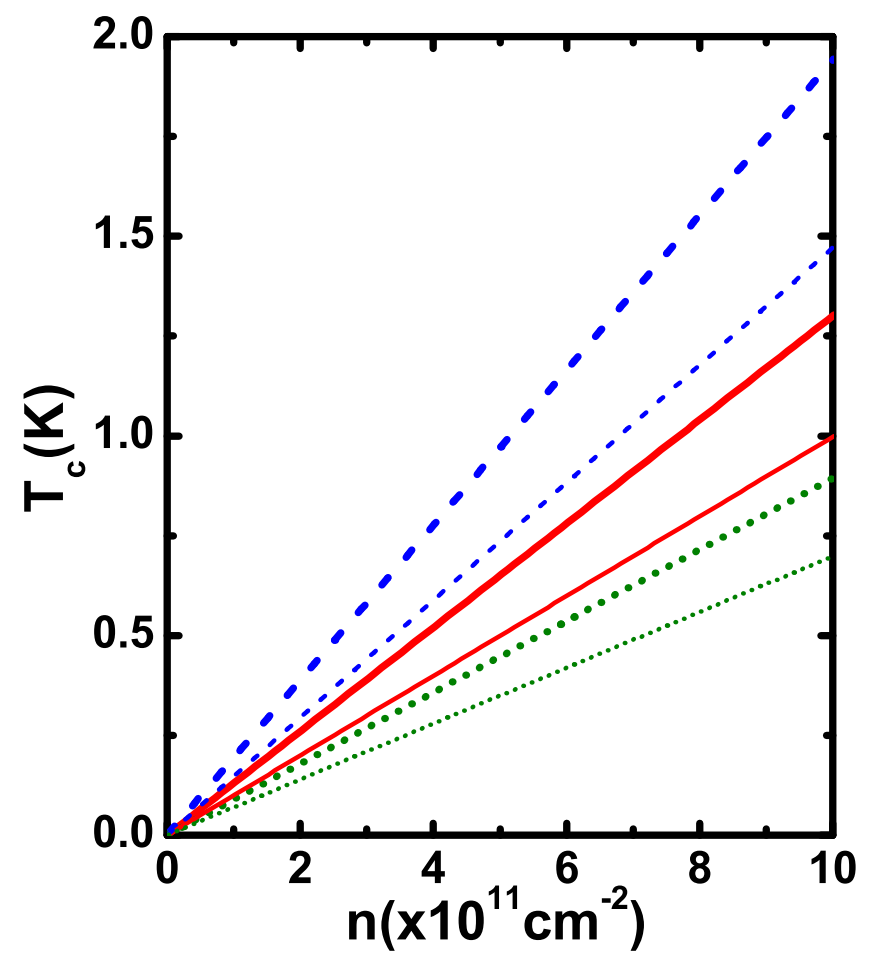

FIG. 4: (Color online) Calculated KT temperature versus magnetoexciton density $n$ at $B=10 \mathrm{~T}$. The solid, dashed, and dotted curves correspond to $D=30,25$, and $35 \mathrm{~nm}$, respectively. Here for comparison, the results with and without inter-LL Coulomb interaction included are given by thick and thin curves, respectively.

absence of inter-LL Coulomb interaction (orange lines), one can find that there is about $20 \% \sim 25 \%$ correction induced by inter-LL Coulomb interaction. By decreasing the magnetic field or the dielectric spacer width, this correction becomes more prominent, which indicates that the inter-LL transition caused by Coulomb interaction is significant in estimating $T_{c}$. When the magnetoexciton mass approximately takes the limit form Eq. (12) at $D \gg r_{B}$, the KT temperature has a promotion (black lines). Especially at relatively small values of $B$ and $D$, this promotion becomes very remarkable and almost reaches 100\%; (iii) Figure 4 shows $T_{c}$ in good approximation linearly increases with increasing magnetoexciton density $n$. This is due to the fact that the denominator in Eq. (15) for the chemical potential and thus the sound velocity, weakly depends on $n$.

\section{CONCLUSION}

In summary, we have theoretically studied the effective mass and KT transition temperature of magnetoexcitons in TIB structure under a strong perpendicular magnetic field. When only intra-LL Coulomb interaction is considered, the effective magnetoexciton mass in TIB structure is four times larger than that in graphene bilayer structure, while the calculated KT temperature is about several Kelvins, same in amplitude as in graphene bilayer and semiconductor bilayer systems. The inclusion of inter-Landau-level Coulomb interaction has been shown to bring about significant corrections to magnetoexciton properties by prominently reducing the magnetoexciton effective mass and promoting the KT temperature.

\section{Acknowledgments}

This work was supported by NSFC under Grants No. 90921003 and No. 10904005, and by the National Basic Research Program of China (973 Program) under Grant No. 2009CB929103.
[1] Y.E. Lozovik and V.I. Yudson, Zh. Eksp. Teor. Fiz. 71, 738 (1976) [Sov. Phys. JETP 44, 389 (1976)].

[2] S.I. Shevchenko, Fiz. Nizk. Temp. 2, 505 (1976) [Sov. J. Low Temp. Phys. 2, 251 (1976)].

[3] D.W. Snoke, Science 298, 1368 (2002).

[4] L.V. Butov, J. Phys.: Condens. Matter 16, R1577 (2004).

[5] V.B. Timofeev and A.V. Gorbunov, J. Appl. Phys. 101, 081708 (2007).

[6] J.P. Eisenstein and A.H. MacDonald, Nature (London) 432, 691 (2004).

[7] C.H. Zhang and Y.N. Joglekar, Phys. Rev. B 77, 233405 (2008).

[8] H. Min, R. Bistrizer, J.-J. Su, and A.H. MacDonald, Phys. Rev. B 78, 121401(R) (2008).

[9] Y.E. Lozovik and A.A. Sokolik, Pis'ma Zh. Eksp. Teor. Fiz. 87, 61 (2008).

[10] A. Iyengar, J.H. Wang, H.A. Fertig, and L. Brey, Phys. Rev. B 75, 125430 (2007).
[11] O.L. Berman, Y.E. Lozovik, and G. Gumbs, Phys. Rev. B 77, 155433 (2008).

[12] O.L. Berman, R.Ya. Kezerashvili, and Y.E. Lozovik, Phys. Rev. B 78, 035135 (2008).

[13] Z.G. Koinov, Phys. Rev. B 79, 073409 (2009).

[14] C. L. Kane and E. J. Mele, Phys. Rev. Lett. 95, 226801 (2005); 95, 146802 (2005).

[15] B.A. Bernevig, T.L. Hughes, and S.-C. Zhang, Science 314, 1757 (2006).

[16] L. Fu and C. L. Kane, Phys. Rev. B 76, 045302 (2007).

[17] M. König, S. Wiedmann, Christoph Brüne, A. Roth, H. Buhmann, L. W. Molenkamp, X.-L. Qi, and S. C. Zhang, Science 318, 766 (2007).

[18] D. Hsieh, D. Qian, L. Wray, Y. Xia, Y. S. Hor, R. J. Cava, and M. Z. Hasan, Nature 452, 970 (2008).

[19] H. J. Zhang, C. X. Liu, X. L. Qi, X. Dai, Z. Fang, and S. C. Zhang, Nature Phys. 5, 438 (2009).

[20] Y. Xia, D. Qian, D. Hsieh, L. Wrayl, A. Pal1, H. Lin, A. 
Bansil, D. Grauer, Y. S. Hor, R. J. Cava, Nat. Phys. 5, 398 (2009).

[21] Y. L. Chen, J. G. Analytis, J. H. Chu, Z. K. Liu, S. K. Mo, X. L. Qi, H. J. Zhang, D. H. Lu, X. Dai, Z. Fang, Science 325, 178 (2009).

[22] M.Z. Hasan and C.L. Kane, Rev. Mod. Phys. 82, 3045 (2010), and references therein.

[23] X.L. Qi and S.C. Zhang, Rev. Mod. Phys. 83, 1057 (2011), and references therein.

[24] J. W. Luo and A. Zunger, Phys. Rev. Lett. 105, 176805 (2010).

[25] Y. S. Hor, A. Richardella, P. Roushan, Y. Xia, J. G. Checkelsky, A. Yazdani, M. Z. Hasan, N. P. Ong, and R. J. Cava, Phys. Rev. B 79, 195208 (2009).

[26] C.-Z. Chang, K. He, L.-L. Wang, X.-C. Ma, M.-H. Liu, Z.-C. Zhang, X. Chen, Y.-Y. Wang, and Q.-K. Xue, Spin 1, 21 (2011).

[27] Z. Li, Y. Qin, Y. Mu, T. Chen, C. Xu, L. He, J. Wan, F. Song, M. Han, G. Wang, J. Nanosci. Nanotechnol. 11, 7042 (2011).

[28] H. Liu and P.D. Ye, Appl. Phys. Lett. 99, 052108 (2011).
[29] R. Valdés Aguilar, A.V. Stier, W. Liu, L.S. Bilbro, D.K. George, N. Bansal, L. Wu, J. Cerne, A.G. Markelz, S. Oh, and N.P. Armitage, Phys. Rev. Lett. 108, 087403 (2012).

[30] H. D. Li, Z. Y. Wang, X. Guo, T. L. Wong, N. Wang, and M. H. Xie, Appl. Phys. Lett. 98, 043104 (2011).

[31] Z. Y. Wang, X. Guo, H. D. Li, T. L. Wong, N. Wang, and M. H. Xie, Appl. Phys. Lett. 99, 023112 (2011).

[32] B. Seradjeh, J.E. Moore, and M. Franz, Phys. Rev. Lett. 103, 066402 (2009).

[33] N. Hao, P. Zhang, and Y. Wang, Phys. Rev. B 84, 155447 (2011).

[34] Y.E. Lozovik and A.A. Sokolik, arXiv: 1111.1176 (2011).

[35] J.M. Kosterlitz and D.J. Thouless, J. Phys. C 6, 1181 (1973).

[36] D.R. Nelson and J.M. Kosterlitz, Phys. Rev. Lett. 39, 1201 (1977).

[37] A.A. Abrikosov, L.P. Gorkov, and I.E. Dzyaloshinski, Methods of Quantum Field Theory in Statistical Physics (Prentice-Hall, Englewood Cliffs, NJ, 1963). 